\title{
A Simplified Preflashover Zone Model of a Fire in a Single Room
}

\section{ANTONIO CRESPO}

Department Fluid Mechanics

E.T.S. Ingenieros Industriales Universidad Politécnica de Madrid C/José Gutiérrez Abascal 2 28006 Madrid, Spain
JULIO HERNANDEZ

Department Mechanics

E.T.S. Ingenieros Industriales

Universidad Nacional de Educación a Distancia

Ciudad Universitaria

28040 Madrid, Spain

\author{
JUAN COLOMBAS \\ Department Fluid Mechanics \\ E.T.S. Ingenieros Industriales \\ Universidad Politécnica de Madrid \\ C/José Gutiérrez Abascal 2, 28006 Madrid, Spain
}

ABSTRACT

Some simplifications have been introduced in the classical models to describe the evolution of fires. They have been applied to single room compartinents, obtaining a significant reduction in the computer time required. The heat equation is solved in solid walls and ceiling by using a global energy conservation equation which utilizes the whole history, from the beginning of the fire, of the temperature at the hot surfaces of the walls. An algorithm has been used to avoid the storage of all the val ues of this surface temperature. Another simplification consists in a sys tematic treatment of the radiative heat transfer between the different elements, which is based on the assumption that they form a closed surface and that the energies reflected from and transmited through the flame are small compared to the energy that it emits; this assumption may be acceptable in many cases of interest. A computer code has been developed to predict the evolution of fires, including the above mentioned sim plifications; although a more complete validation of the code is necessary, preliminary comparisons with other codes and experiments have been satisfactory.

\section{INTRODUCTION}

Zone models have been used extensively to calculate fire evolution in buildings, and have been evaluated and compared in several review papers on this subject 1,2 . One of the main difficulties of these models is the enormous number of zones appearing when they are extended from a single room to whole buildings, which may result in large computer capacity requirements; consequently, any simplification in the treatment of any of the zones is desirable. On the other hand, there is a large uncertainty in the validity of the empirical expressions and coefficients which are used in these models, what may introduce errors which are much larger than those due to some mathematical simplifications, such as those that will be proposed here.

In this work, a simplified approach has been used to model the radiative heat transfer between the different elenents of a single room, and to solve the heat equation in solid elements. To validate these simplifications, a computer code for a single room, based on the model of Harvard 3,4 , has been developed. Although patterned after the Harvard model, the code presented in this work is new, and not a modified version of the Harvard program. It has been implemented in BASIC language in a HP-9816 microcomputer, and solves the system of finite-difference equa- 
tions by using a Gauss-Seidel method.

A general description of the zone model is made in the next section, and in the following two sections the most relevant features of the model, related with the methods used to treat the radiative heat transfer and the heat conduction in the solids, are presented. Finally, a comparison is made of the results of the proposed code with those of the Fifth Harvard Code 4 and the FAST code 5.

\section{GENERAL DESCRIPTION OF THE MODEL}

The model is based on the classical models utilized to describe fires in single room compartments 3,4 , and consists of a burning object, a plume, a hot layer, and a single opening through which there can be flow in both ways, in and out, simultaneously. There is also an inert object, which is heated by radiation from the flame, the hot layer and the walls, and whose surface temperature is calculated continuously during the process.

The flow through the opening is calculated by using classical expres sions ${ }^{4}$, and assuming that in the outside there is ambient pressure.

The flame is supposed to have the shape of a hollow cone of given thickness and apex angle. The radius, as a function of time, is also given as an input to the code. When there is enough oxygen, the apex angle is supposed to have a fixed value, and for oxigen starvation conditions the tangent of this angle increases proportionally to the effective fuel-oxygen ratio.

The mass loss rate of the burning object is calculated from the ram tio of the heat transferred to its surface and the heat of pyrolisis. In this model it is assumed that this heat transfer is mainiy due to flame radiation. The mass burning rate is supposed to be a given fraction of the mass loss rate if there is not oxygen starvation.

The hot layer has uniform temperature, as well as the surface of the walls in contact with it and the ceiling. The lower layer and the walls in contact with it and the floor are supposed to be at the ambient temper ature. The coefficients to calculate convective heat transfer have been taken from the literature 3,4 .

\section{HEAT TRANSFER BY RADIATION}

To calculate the radiative heat transfers among the walls, floor, flame and ceiling, it has been used a simplified version of the following general equation, where the flame is considered as a surface:

$\sum_{j}\left[\delta_{k j}-\left(1-\varepsilon_{j}\right) \tau_{k j} F_{k j}\right] \frac{\dot{E}_{j}}{\varepsilon_{j} A_{j}}=\Sigma_{j}\left[\delta_{k j}-\tau_{k j} F_{k j}\right] \sigma T_{j}^{4}-\overline{g_{C} S_{k}} \frac{\sigma T_{C}^{4}}{A_{k}}$,

where the subscripts $k$ and $j$ stand for the upper surface, lower surface, flame and inert object (Fig. 1), and $\dot{E}_{j}$ is the net heat emitted by radiation by surface $j$; the last term corresponds to the heat transfer between the hot layer and surface $k$, being $\overline{g_{C} s_{k}}$ the corresponding volume-surface exchange coefficient. $\tau_{k j}$ denotes the transmittance through the hot layer between $k$ and $j$ elements, 
$\tau_{k j}=1-\alpha_{k j} \simeq 1-\exp \left(-k_{c} L_{k j}\right)$,

where $L_{k j}$ is the mean length through the hot layer between surfaces $k$ and $j$, and $k_{c}$ the absorptivity of the hot gases. The flame, that is supposed to radiate as a gray body, is considered to be small enough that both transmission and reflection processes can be treated simultaneously by using a single coefficient $1-\varepsilon_{f}$.

If it is assumed that, for the flame, $F_{k k}=0$, and that walls, ceiling, floor and inert object are black surfaces $\left(\varepsilon_{j}=1\right)$, the corresponding Eq. (1) for the flame can be simplified to

$\frac{\dot{E_{f}}}{E_{f} A_{f}}=\sum_{j}\left[\delta_{f j}-F_{f j}\left(1-\alpha_{f j}\right)\right] \sigma T_{j}^{4}-\sum_{j} F_{f j} \alpha_{f j} \sigma_{C}^{4}$,

where the subscript $f$ stands for flame and the last term is derived by assuming that all the elements form a closed surface, neglecting the effect of the opening, and that there is only one hot layer. Furthermore, if temperatures of the other elements are small enough compared to the flame temperature, it can be written

$\dot{E}_{f}=\varepsilon_{f} A_{f} \sigma T_{f}^{4}$.

Taking into account Eq. (4), a simplified version of Eq. (1) can be deduced for any element $k$ (even for $f$ ):

$\frac{\dot{E}_{k}}{\varepsilon_{k} A_{k}}=\sum_{j}\left[\delta_{k j}-F_{k j}\left(1-\alpha_{k j}\right) \varepsilon_{j}\right] \sigma T_{j}^{4}-\sum_{j} F_{k j} \alpha_{k j} \sigma T_{c}^{4}$.

An equivalent equation can be obtained by assuming that the flame can not reflect.

upper surface

lower surface

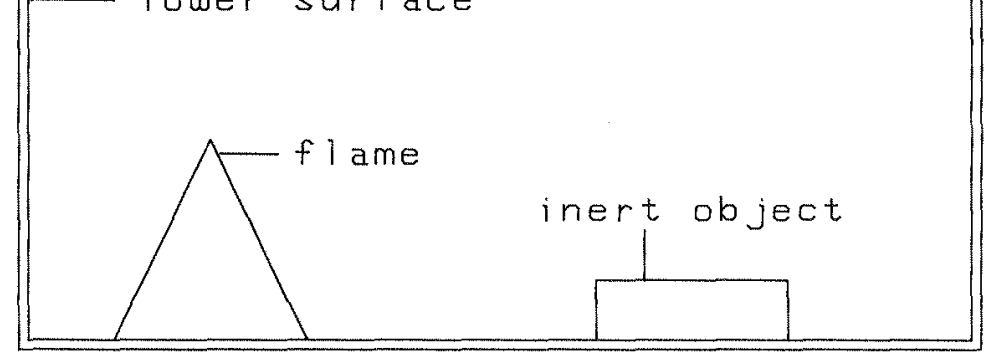

FIGURE 1. Schematic to model the radiative processes. 
The effective emissivity of the hot layer, that is supposed to be gray, is taken from Mitler 4 . The lower layer is supposed to be transparent. Simplified expressions, based mostly in the fact that the inert object and the flame are small, are chosen for the view factors.

The net influx of radiation to the hot layer is obtained from a global balance applied to the above mentioned closed surface, and neglecting the energy transmitted through the flame because of its small volume when compared with the volume of the hot layer, and that going out vents,

$\dot{\mathrm{E}}_{\mathrm{C}}=-\sum_{\mathrm{k}} \dot{\mathrm{E}}_{\mathrm{K}}$

The above procedure does not allow one to calculate the energy trans ferred by radiation to the burning object, that is obtained from an expression taken from Emmons ${ }^{3}$.

\section{SURFACE TEMPERATURE OF THE SOLIDS}

To calculate the temperature at the surface of the hot walls, ceiling and inert object, it is necessary to solve the heat equation, imposing boundary conditions at both sides of the element. It is proposed to simplify this problem by assuming that the thickness of the solid element is much larger than the depth of the heated region, so that the outer boundary condition is that at infinity: it has ambient temperature there.

The temperature at the hot side of the wall is calculated from a glo bal energy conservation equation for the solid,

$\frac{\mathrm{dep}}{\mathrm{dt}}=\dot{Q}$

which involves the heat stored in the wall during the process from its initiation; this magnitude can be expressed as

$e_{p}=\sqrt{\rho_{s} c_{s} k_{s} / \pi} s_{0}^{t} \frac{T_{i}\left(t^{\prime}\right)-T_{a}}{\sqrt{t-t^{\prime}}} d t^{\prime}$.

The integrand is a function of the previous history of the temperature of the inner side of the solid. To avoid storing all the previous values of this temperature, an algorithm is used to calculate the heat accumulated in the solid. This algorithm is based on assuming linear variation of this wall temperature with time, during time intervals small enough to re duce the error below a given value (Fig. 2). For example, during the first interval it is obtained that

$e_{p}=\sqrt{\rho_{s} c_{s} k_{s} / \pi} \frac{4}{3}\left(T_{i}(t)-T_{a}\right) \sqrt{t}$,

and so on for other time intervals. Therefore, for each time interval a re lationship between $e_{p}$ and the temperature of the inner side of the wall can be obtained. From this relationship and $\mathrm{Eq} .(7), \mathrm{T}_{i}$ can be determined as a function of time.

The time intervals turn out to be large enough that only about one fiftieth of the calculated temperature values need to be stored.

The proposed method does not work for thermally thin walls beyond the time required for the thermal wave to traverse the wall. In many 


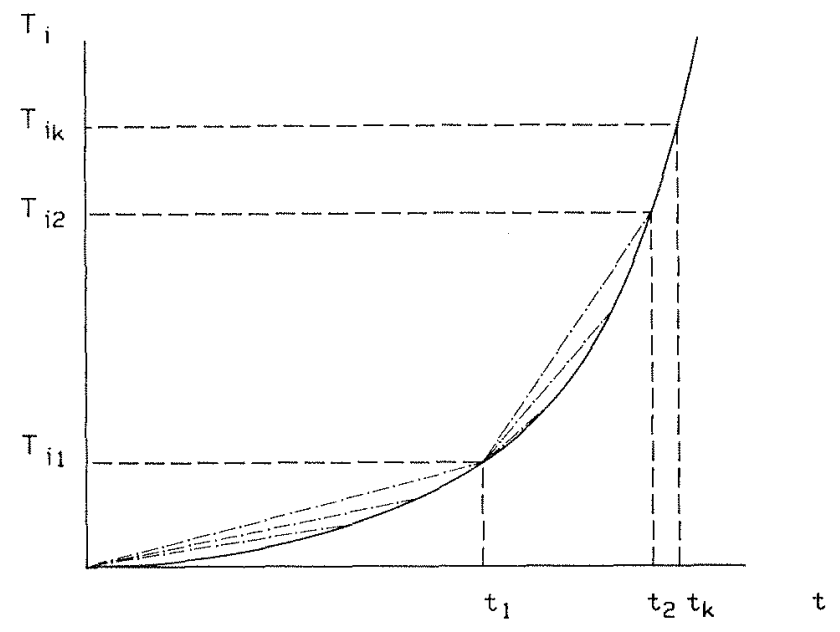

FIGURE 2. Representation of the surface temperature of solids to calculate the integral of $\mathrm{Eq}$. (8).

cases, this time is long compared to the typical duration of the preflashover fire period.

The inner solid temperature has also been calculated by solving the one-dimensional heat equation in the solid, and the results obtained are very similar to those obtained by using the algorithm described in this section, provided that a small enough grid spacing and a short time inter val are chosen. To do this comparison, the code developed was modified by substituting the proposed algorithm by an explicit method to solve the heat equation.

In Fig. 3 the CPU time required by the proposed code to solve a typi cal problem of a fire in a single room is presented, for two cases, as a function of the physical time. In the first case, the heat equation is solved using an explicit method; in the second one, the alternative algorithm proposed here is used. It may be seen that the proposed method may represent an interesting contribution in reducing the computing time.

The proposed method to calculate the temperature of the hot surfaces of the solids has also the advantage that it has not the instability which appears when solving the heat equation by an explicit method; in this last case there would be a limitation on the minimum size of the spa tial grid or on the maximum interval of time. If an implicit method were used to solve the heat equation, there would not be this type of instabil ity, but much more cPU time would be consumed, and the saving resulting from the proposed method would be much more important than that represented in Fig. 3 . 


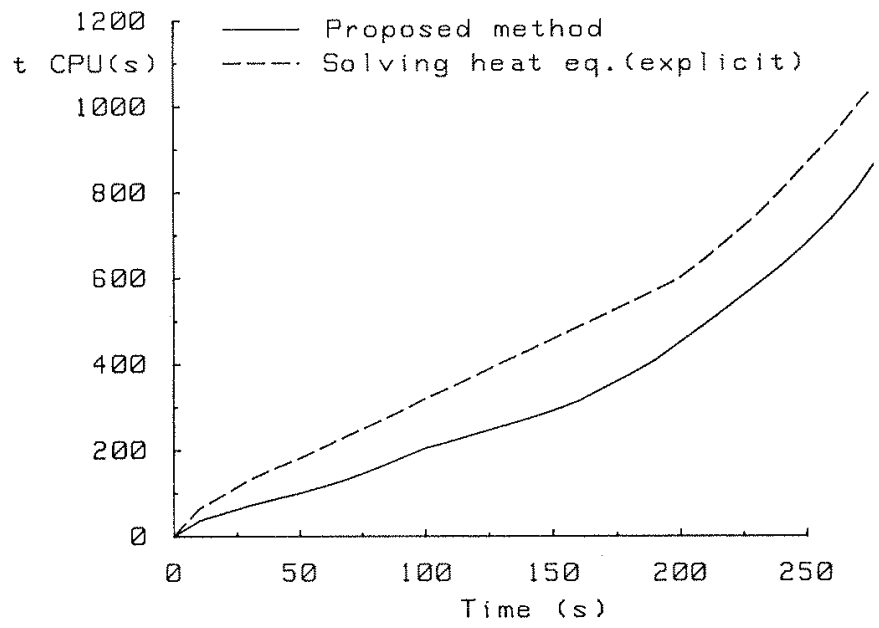

FIGURE 3. CPU time required by the proposed code to solve a test case, as a function of the physical time, by using two different methods to treat the heat conduction in solids.

\section{COMPARISON WITH OTHER MODELS}

The proposed code has been applied to a case solved by Mitler ${ }^{4}$ with the Fifth Harvard Computer Code. In Figs. 4 an 5 are presented the results obtained for the hot layer depth and temperature calculated with our code, and they are compared with the results given by Mitler 4 .

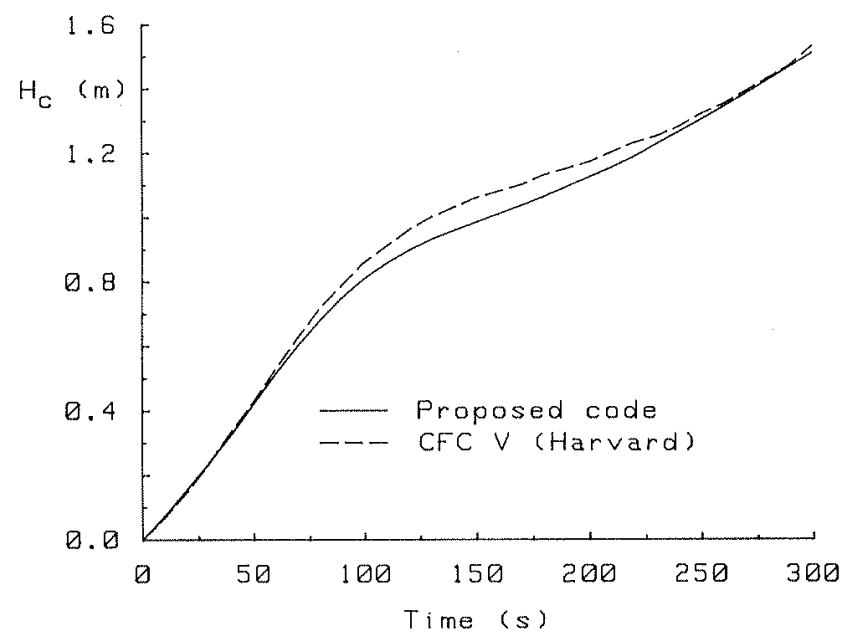

FIGURE 4. Depth of the hot layer. Comparison between results of the proposed code and CFC $V$ Harvard code. 


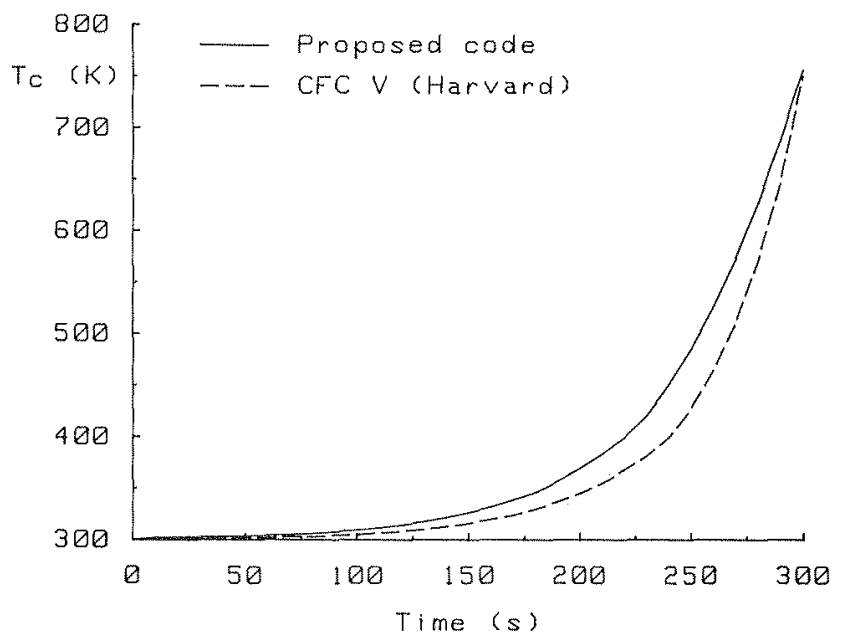

FIGURE 5. Temperature of the hot layer. Comparison between results of the proposed code and CFC V harvard code.

A comparison has also been carried out with a case solved by Walton et al. 5 with the FAST code. The conditions of the case for which results are presented in that reference can not be exactly simulated with our code, mainly because there are several rooms and openings. The single opening of the model presented here can be made equivalent to the combina tion of the openings of the room where the fire starts in the FAST code. The results of this multiroom case can be compared with ours if the time from fire start is small enough that ambient pressure does not differ much from the pressure in the adjacent rooms, and the hot layer in those rooms does not go below the si11. There is a datum satisfying the above condition, which corresponds to 60 seconds from start; the comparison is very good for the depth and temperature of the hot layer, but the proposed code predicts a larger temperature of the upper walls and ceiling.

\section{ACKNOWLEDGEMENT}

We are very grateful to the 'Instituto Tecnológico de Seguridad de MAPFRE' (ITSEMAP), Spain, for its help in the realization of this work.

\section{REFERENCES}

1. Jones, W. W., "A Review of Compartment Fire Models," National Bureau of Standards, NBSIR 83-2684, 1983.

2. Cox, G., "Simulating Fires in Buildings by Computer - The State of the Art," 10th International Association of Forensic Sciences Meeting, Oxford, 1984.

3. Emmons, H. W., "The Calculation of a Fire in a Large Building," Journal of Heat Transfer, 105, pp. 141-158, 1983. 
4. Mitler, H. E. and Emmons, H. W., "Documentation for the Fifth Harvard Computer Fire Code," Home Fire Project Technical report 45, Harvard University, Cambridge, MA, 1981.

5. Walton, W., Baer, S. and Jones, W., "User's Guide for FAST," National Bureau of Standards, NBSIR 85-3284, 1985.

\section{NOMENCLATURE}

$A_{k} \quad$ area of surface $k$

$c_{s}$ specific heat of solids

$\dot{\mathrm{E}}_{\mathrm{k}}^{\mathrm{S}}$ net heat rate emitted by radiation by surface $\mathrm{k}$

$\dot{\mathrm{E}}_{c}$ net rate of energy absorbed by radiation by the hot layer

$e_{p}$ energy per unit of area stored in the solids

$F_{k j}$ view factor of surface $k$ as seen by surface $j$

$\mathrm{H}_{\mathrm{C}}$ depth of the hot layer

$k_{s}$ thermal conductivity of solids

$L_{k j}$ mean length through the hot layer between surfaces $k$ and $j$

$\dot{Q}$ net influx of energy per unit time to solid surfaces

$\mathrm{T}_{j}$ temperature of surface $j$

$\mathrm{T}_{\mathrm{a}}$ ambient temperature

$\mathrm{T}_{\mathrm{C}}$ temperature of the hot layer

$\mathrm{T}_{j} \quad$ temperature of the inner hot side of solids

$t, t^{\prime}$ time

$\alpha_{k j}$ absorption coefficient

$\delta_{k j} \quad$ Kronecker delta

$\varepsilon_{k}$ emissivity of surface $k$

${ }^{k_{c}}$ absortivity of the hot gases $\left(\mathrm{m}^{-1}\right)$

$P_{S}$ mass density of solids

o Stefan-Boltzmann constant

$\tau_{k j}$ transmittance through the hot layer between elements $k$ and $j$ 